\title{
e-Migrinter
}

$6 \mid 2010$

Rroms \& Gens du Voyage

\section{« Migrants, santé et expériences de la discrimination "}

Compte rendu du colloque organisé à la MSHS de Poitiers le 12 mars 2010

\section{Anne-Cécile Hoyez}

\section{OpenEdition}

\section{Journals}

\section{Édition électronique}

URL : https://journals.openedition.org/e-migrinter/1313

DOI : 10.4000/e-migrinter.1313

ISSN : 1961-9685

\section{Éditeur}

UMR 7301 - Migrinter

\section{Édition imprimée}

Date de publication : 15 octobre 2010

Pagination : 86-93

ISSN : 1961-9685

\section{Référence électronique}

Anne-Cécile Hoyez, « " Migrants, santé et expériences de la discrimination » », e-Migrinter [En ligne], 6 | 2010, mis en ligne le 22 août 2019, consulté le 20 mai 2021. URL : http://journals.openedition.org/emigrinter/1313 ; DOI : https://doi.org/10.4000/e-migrinter.1313 


\section{" Migrants, santé et expériences de la discrimination ", Colloque organisé à la MSHS de Poitiers le 12 mars 2010}

Compte rendue par Anne-Cécile Hoyez

e colloque, co-organisé par
Migrinter (CNRS/Université
de Poitiers), la Mairie de
Poitiers et l'ACSÉ (Agence
nationale pour la Cohésion Sociale
et l'Égalité des chances), avait
pour but de rassembler chercheurs
et professionnels autour de la
question des discriminations dans
l'accès aux soins. Près de 70
personnes se sont inscrites pour y
assister, et 12 chercheurs et
professionnels de la santé et du
social ont exposé leurs travaux de
recherches, leurs expériences
professionnelles et leurs
questionnements.

Introduction et contexte

L'an passé, à la même époque, un colloque du même format portait sur les politiques migratoires et les luttes contre les discriminations. Les discussions ont été riches en informations et en débats, et ont révélé la complexité du champ d'investigation: complexité des types de discriminations, des échelles des discriminations, des perceptions et des vécus des discriminations, et, finalement, la complexité des interprétations des discriminations, voire leurs pièges.

Dans la continuité de ces réflexions, et dans la continuité d'échanges qui se sont institués à la suite du colloque de 2009 , nous avons décliné, cette année, la question des discriminations à un domaine de la vie en société : la santé (considérant que la santé n'est pas qu'une affaire médicale mais aussi une question globale, touchant au politique, au social voire au culturel).

L'inscription de cette question dans ce colloque vient également de préoccupations scientifiques et professionnelles qui nous interrogent de plus en plus. On remarque en effet, d'un côté, un intérêt de plus en plus marqué dans différents champs de la recherche (sciences sociales, santé publique) pour l'analyse conjointe des relations «triadiques» entre différents domaines complexes (migrations, santé, processus de discriminations) et des relations verticales qui régissent cette triade (processus institutionnels, politiques, idéologiques qui les orientent, à différentes échelles). D'un autre côté, on voit se concentrer des questions de plus en plus complexes pour les professionnels de la santé et du social qui doivent, en plus de s'adapter à des changements (politiques, économiques) constants, négo- 
cier, contourner, et composer pour proposer une offre de soins efficiente et efficace aux migrants, qu'ils soient primo-arrivants ou installés depuis plus longtemps.

Cette journée a donc été l'occasion de mettre en lien plusieurs de ces approches, de faire dialoguer des chercheurs avec des professionnels de la santé et du social, pour tenter de faire un état des lieux sur la question et de faire avancer la réflexion conjointement.

Nous avons eu la chance de pouvoir donner la parole et d'échanger avec des collègues britanniques et allemands, pour qui la question peut se poser différemment, compte-tenu des différents contextes historiques, politiques et sociaux de l'immigration et de la santé.

Les présentations, qui sont résumées ci-après, ne sont pas toutes axées directement sur les questions de discriminations, mais elles tournent autour, en abordant tout autant le lien entre migrations et santé.

\section{Résumé des interventions}

Durant la matinée, nous avons pu explorer ce qui se passe chez nos voisins britanniques et allemands, à travers la présentation de plusieurs collègues venus exposer leurs différents travaux.

Robin Goodwin (Brunel University) est d'abord intervenu sur la question des " nouveaux » migrants polonais, ceux dont les parcours migratoires sont constitués d'allers-retours fréquents entre le RoyaumeUni et la Pologne. Ces mobilités impliquent des rapports différenciés aux systèmes de soins des deux pays, et par conséquent on observe de nouvelles pratiques de soins émerger parmi ces migrants. Tout d'abord, devant l'accroissement de la prévalence des maladies sexuellement transmissibles et en particulier du VIH-Sida chez les migrants polonais, le gouvernement polonais a mis en place depuis le début des années 2000 des programmes spéciaux pour informer et prévenir des risques liés à la transmission de ces maladies. Les migrants polonais sont donc considérés comme des populations à risque par leur gouvernement et sont la cible d'actions de santé publique spécifique. D’un autre côté, l'état Britannique, via son système de santé (le NHS : National Health System), considère ces migrants comme des minorités ayant accès, comme les autres, au NHS, mais sans critère de vigilance sanitaire spécifique. L'étude de R. Goodwin se place du côté des migrants et de leur vécu des discriminations au sein de ce système. D'une part, une enquête révèle que les migrants polonais ont conscience des différences de traitement dont ils font l'objet dans les deux pays et de toutes les conséquences que cela peut avoir pour eux dans leur accès aux soins (population à risque en Pologne / population minoritaire en Grande-Bretagne). Cependant, s'ils font plus facilement confiance au niveau de formation des médecins britanniques et à leur indépendance (notamment par rapport aux laboratoires pharmaceutiques), ils subissent les effets systémiques comme des discriminations: problèmes dans l'orientation dans le système de soins, et difficultés à exprimer leur souffrance et symptômes face à des médecins dont le temps de consultation est très réduit, et dont les représentations des migrants peuvent être erronées.

Nasir Warfa (QMUL) a présenté les résultats d'une étude sur les réfugiés somaliens à Londres, explicitant le lien entre santé mentale et psychologique et conditions pré- et post-migratoires. L'accès aux soins, dans le système britannique, est lié au lieu de résidence: le parcours de soins est borné aux visites chez les généralistes et les hôpitaux du quartier où réside la personne. Cette disposition est problématique pour les patients mobiles; elle l'est encore plus chez les populations pour qui les conditions d'obtention d'un logement et la stabilité de résidence est dépendante des ressources économiques et des statuts administratifs, c'est-à-dire pour les minorités ethniques et 
les migrants. Warfa a montré que les réfugiés somaliens entrent dans ces catégories de personnes précaires: ils sont contraints à une mobilité qui les maintient dans des quartiers et des logements de mauvaise qualité. Hormis les questions de l'incidence de ces mobilités sur la santé mentale (instabilité et précarité sont des déterminants importants de l'état de santé) et des conditions de logement (insalubrité et insécurité résidentielle), les réfugiés somaliens connaissent de sérieuses entraves dans leur suivi médical, car ils se trouvent obligés de changer en même temps de résidence, de médecin et d'hôpital de référence. À ces conditions post-migratoires se conjuguent les conditions pré-migratoires, qui avaient déjà largement agit sur l'état de santé des réfugiés: l'exposition à des évènements traumatiques en Somalie ont provoqué des désordres psychiatriques importants chez ces réfugiés, nécessitant un suivi régulier et long qu'il est difficile de maintenir en raison des conditions post-migratoires. Le stress pré- et post-migratoire, la résurgence d'évènements traumatiques, les expériences d'acculturation difficiles, les expériences du racisme et de la discrimination, les expériences d'autres problèmes dans le pays d'accueil conduisent au développement de pathologies psychiatriques importantes chez les réfugiés somaliens. L'enquête de Warfa n'a pas révélé de problèmes de discriminations directes dans l'accès aux soins (les discriminations sont par contre plus marquées dans le domaine de l'emploi et du travail). En revanche, cette enquête souligne l'ensemble des problèmes liés aux impacts des conditions de vie, dans le pays de départ et dans le pays d'accueil, et aux problèmes inhérents au système de santé publique (critère de résidence, formation et qualification des médecins dans le cadre de la pratique auprès de populations souffrants de pathologies liées à un ensemble de conditions particulières).

Estelle Carde (sociologue, médecin de santé publique) a présenté ses travaux sur les discriminations selon l'origine dans l'accès aux soins, qu'elle a menés en France métropolitaine et d'outre-mer (en Guyane). Sur l'ensemble de ces terrains, des discriminations sont opérées à l'encontre des étrangers, lors de leur accès aux droits à une couverture maladie, par des professionnels qui appliquent de façon restrictive textes de loi et réglementations (par exemple en exigeant des justificatifs par excès). Ces pratiques discriminatoires font écho à un contexte politique national caractérisé depuis les années 1970-80 par d'une part des politiques migratoires qui restreignent les droits sociaux des étrangers (afin de limiter «l'appel d'air » qu'induirait un système social généreux) et d'autre part des préoccupations concernant le niveau des dépenses de santé. Les professionnels «répercutent » «l'esprit» de ces politiques, qui tendent à associer la figure sociale de l'étranger à celle du profiteur, dans leurs pratiques. La comparaison Métropole / Guyane révèle que ces pratiques discriminatoires sont plus répandues en Guyane, territoire français soumis aux mêmes textes de loi et aux mêmes réglementations qu'en métropole, mais où l'immigration est de fait beaucoup plus importante, conduisant les professionnels à considérer que les spécificités de leur territoire justifient d'y adapter la législation nationale dans un sens restrictif. Par ailleurs, en Guyane, l'usage banal des dénominations ethno-raciales donne à voir, de façon plus explicite qu'en France métropolitaine, la façon dont des discriminations sont opérées à l'encontre non seulement des étrangers mais aussi des minorités ethnoraciales. Est alors en cause non plus l'origine objectivement définie par la nationalité, mais l'origine socialement perçue. Enfin, l'étude guyanaise révèle la juxtaposition des inégalités sociales dans l'accès aux soins, les mêmes étant souvent à la fois, d'une part, victimes de discriminations selon l'origine et d'autre part, défavorisés dans leur accès aux soins du fait de leur zone d'habitation (dans l'Intérieur du département, où l'offre de soins est dispersée) et de leur précarité socio-économique (les dysfonctionnements dans l'accès 
aux droits affectant spécifiquement ceux dont relèvent les plus précaires).

Les communications présentées au cours de cette matinée ont eu pour intérêt de comparer les expériences des discriminations dans différents pays et territoires. Elles ont ainsi pu mettre en lumière les différents niveaux qui interviennent dans l'expérience, la perception et la fabrication des discriminations dans le domaine de l'accès aux soins (du point de vue législatif comme du point de vue social). Les processus de discriminations qui ont été décrits et analysés sont en grande partie générés par les effets délétères des manifestations de processus inégalitaires plus larges: inégalités territoriales dans l'accès aux soins, inégalités socioéconomiques au sein de certains groupes de populations, inégalités ethnoraciales engendrées par différents processus de représentations sociales. Ces communications ont laissé des pistes de discussion ouvertes pour la suite: par exemple, l'importance donnée au milieu urbain dans les études de cas présentées invite à s'interroger sur ce qui se passe en milieu rural, et l'importance donnée aux processus macro-sociologiques et politiques invite à réfléchir sur la mise en place d'outils spéciaux, dans les services publics de santé, pouvant permettre une pratique facilitée auprès des publics migrants (le cas de la traduction en premier lieu).

L'après-midi a été plus axée sur le contexte national français et le contexte local poitevin, avec des interventions analysant les pratiques de soins, du point de vue des professionnels et des patients migrants, et l'état de santé des populations migrantes.

Une première session sur les contextes nationaux et locaux des pratiques de soins et des droits à la santé des migrants a permis d'interroger les deux niveaux de l'organisation des soins à destination des migrants.
Estelle d'Halluin a commencé par replacer les questions de santé au sein des procédures de régularisation et de demande d'asile. En effet, l'expertise des médecins est de plus en plus convoquée (depuis la loi de 1998) de façon formelle (désignation des experts médicaux dans les procédures de régularisation) ou plutôt informelle (médecins engagés auprès de leurs patients migrants qui vont certifier les séquelles physiques ou psychologiques dans le cadre de la procédure d'asile). La place de l'expertise est donc croissante, et ne se limite pas qu'aux médecins exerçant en libéral, puisque de nombreuses associations se sont saisi de cette question (COMEDE, Primo Levi). Avant 1998, la régularisation pour soins, justifiée pour des raisons de santé, était délivrée au cas par cas, laissant alors la « raison humanitaire » guider les décisions : le droit à être protégé dépendait de la façon dont on considérait les corps souffrants (voir les travaux de D. Fassin). Après la loi de 1998 le droit à être régularisé pour soins a été édicté, provoquant alors l'accroissement des demandes auprès des médecins de personnes souhaitant faire valoir leur droit. Cependant, en contexte de maîtrise des flux migratoires, ce droit a été restreint dans sa mise en œuvre, et restreint directement par les agents de l'Etat chargés d'instruire les dossiers. De manière parallèle, en ce qui concerne l'expertise plus informelle convoquée dans la procédure d'asile, la réception des certificats relatifs aux séquelles physiques et psychiques des exilés se caractérise par une pluralité et une opacité des modes d'appréciation. De la même façon que l'a exposé précédemment E. Carde, on a vu alors se développer des processus que l'on pourrait qualifier de discriminatoires, tant les inégalités devant le droit à la régularisation pour soins d'une part, et le droit d'asile, d'autre part, sont fonction du capital social et culturel des migrants et demandeurs d'asile tout autant que des positions des juges chargés d'apprécier leur requête. Ces dysfonctionnements ont provoqué un important mouvement de réflexion chez les 
médecins et les associations impliquées dans l'accès aux droits et aux soins des migrants, car ils posent des questions éthiques, politiques et pratiques. Pour exemple, dans le domaine de l'asile, l'avis des médecinsexperts pouvant être utilisé à des fins de sélection des demandeurs d'asile et le certificat médical prenant une place croissante dans la procédure d'asile et la parole de l'expert remplaçant ou effaçant la parole du demandeur d'asile amène les professionnels de la santé à s'interroger sur leur pratique professionnelle (de plus en plus sollicités sur un terrain administratif alors qu'ils entendaient avant tout délivrer des soins), éthique et politique (leur expertise pouvait renforcer une interprétation restrictive de la convention de Genève sur le droit à la protection : les requérants ayant subi, et non uniquement craint, des persécutions devenant prioritaires dans le traitement de la demande d'asile).

A la suite de cette présentation, Myriam Hachimi-Alaoui a poursuivi sur la question des professionnels de santé, mais cette fois en analysant leurs pratiques quotidiennes face à l'immigration irrégulière. Reprenant des travaux menés en 2008 en France, en Grande-Bretagne et en Suisse, cette présentation a interrogé l'articulation entre politiques nationales et pratiques locales dans la prise en charge des migrants en situation irrégulière. Dans les trois pays de l'enquête, le climat politique est globalement défavorable à l'accès aux soins des migrants irréguliers, et les dernières dispositions règlementaires viennent encore complexifier leurs conditions d'accès aux soins. Même si des dispositifs nationaux de prise en charge existent -très différents d'un pays à l'autre, ils sont réservés soit à des pathologies particulières, soit à des situations exceptionnelles. Dans ces contextes, les personnels de santé voient leurs pratiques professionnelles être constamment reformulées en fonction de l'évolution des dispositifs juridiques et administratifs. A partir d'entretiens menés avec ces professionnels et avec les professionnels du social (assistantes sociales des PASS par exemple), l'étude souligne à quel point leurs pratiques conduisent à des stratégies pour s'adapter aux changements ou les contourner, ou bien à des conflits. Tous ces changements, aussi bien du côté des politiques nationales que des pratiques locales poussent à s'interroger sur les transformations actuelles des systèmes de santé face à l'évolution des politiques migratoires dans les pays européens.

Pour illustrer ces contextes locaux et les adaptations locales telles qu'elles sont pratiquées sur le terrain, le Dr Philippe Aubry (PH au CHU de Poitiers et au Relai Georges Charbonnier) et Karine Bonnifait (assistante sociale à la PASS de Poitiers) nous ont présenté leurs actions pour permettre l'accès aux droits et aux soins de toute population dont également la population migrante. Le dispositif dont il s'agit date du début des années 1990, où il s'organisait alors autour de l'action locale de quelques professionnels de la santé et du social; il a ensuite été renforcé et formalisé suite à la loi du 29/07/1998 relative à la lutte contre l'exclusion. Les réponses des pouvoirs publics à l'exclusion du système de droit commun pour les soins a donc été de créer les PASS (Permanences d'Accès aux Soins de Santé), la CMU (Couverture Médicale Universelle) base et complémentaire, l'AME (Aide Médicale d'Etat), la PSU (Prise en charge des Soins Urgents). A Poitiers, s'est donc mise en place une PASS interne, intervenant au sein du CHU, et une PASS externe au Relais Georges Charbonnier, dont les missions sont l'accueil (des publics les plus défavorisés, migrants ou non), la présence sociale, la réponse aux besoins de soins, la cohésion et la cohérence entre les différents services du médical et du social. Le personnel composant la PASS externe regroupe 3 assistantes sociales (2,3 ETP), 2 médecins, 1 infirmier (0,5 ETP), 1 pharmacien $(0,1$ ETP), un interne en médecine générale. Une analyse des activités médicales montre que le nombre de consultations est en augmentation constante depuis 2005 
(+62\% entre 2007 et 2009), et que la file active des patients croît tout aussi régulièrement (+44\% entre 2007 et 2009). Les motifs de consultation les plus fréquents concernent les visites simples, le suivi, l'information à la santé. Mais les demandes les plus pressantes et les plus nombreuses concernent aujourd'hui la médecine préventive et l'accompagnement vers le droit commun ( $+86 \%$ entre 2008 et 2009), ce dernier dispositif étant favorisé par l'interprétariat. Le Relai Georges Charbonnier évolue suivant un réseau de partenaires médicaux dense à l'échelle locale (de la médecine libérale aux institutions publiques locales, telles la CPAM ou les PMI). A court terme, ce réseau pourrait être densifié avec la création d'une PASS dentaire. Une deuxième session visait à présenter les travaux récents sur l'état de santé des migrants en France et à les lier avec des exemples de mise en ouvre d'actions locales ciblant des pathologies particulières.

Paul Dourgnon a entamé la session en synthétisant l'étude menée par l'IRDES en 2008. Les objectifs de l'étude menée par les chercheurs de l'IRDES avaient pour priorité d'étudier les liens entre nationalité, migration et état de santé, en mesurant les inégalités de santé, à l'aide de plusieurs indicateurs de santé, liées au statut (français de naissance, immigré français par naturalisation, immigré étranger) et au pays de naissance, de distinguer l'influence directe de la migration et de la nationalité sur la santé, de son influence indirecte passant par la situation socioéconomique et les conditions de travail des immigrés en France, et d'étudier l'hypothèse d'un effet à long terme des conditions économiques et sanitaires du pays d'origine. L'étude souligne que les immigrés naturalisés et étrangers déclarent un plus mauvais état de santé ressenti, en partie en raison de leur situation économique et sociale moins favorable et de conditions de travail plus pénibles. Mais cette différence subsiste pour les immigrés étrangers comme naturalisés après prise en compte du SES (Statut Economique et Social) et des conditions de travail : on peut ici faire l'hypothèse d'effets délétères de la migration (déracinement, isolement, discriminations). Il existe d'autre part une hétérogénéité selon le pays d'origine : après contrôle par le SES, meilleur état de santé des immigrés originaires de l'Europe du Nord, , plus mauvais état de santé perçu des immigrés originaires de l'Europe du Sud, un plus mauvais état de santé des immigrés originaires de l'Europe Centrale et de Turquie, expliqué par leur SES, et un plus mauvais état de santé perçu et meilleur état de santé selon les 2 autres indicateurs pour les immigrés originaires du Maghreb et d'Asie. L'impact du contexte économique et social du pays d'origine a également une influence. Les personnes issues des pays présentant les niveaux de richesse les plus bas comme les plus hauts ont un meilleur état de santé. On pourrait lire ici les effets à long terme des conditions économiques et sociales favorables des pays riches (dont un bon système de santé) du pays d'origine, conjugués aux effets de sélection de migrants en bonne santé des pays plus pauvres.

Ensuite, Brigitte Berthier-Wilson est intervenue sur la question de la prévention du saturnisme infantile au sein de la population Africaine de la Vienne. Le saturnisme infantile est une intoxication par le plomb des enfants (plomb issu des céruses, carburants, canalisations, sols pollués, ferraillage, cosmétique, bijoux, etc..). Il touche en particulier les enfants de moins de 6 ans, atteints alors de symptômes inconstants, de troubles réversibles (anémie, troubles digestifs) ou irréversibles (atteinte du système nerveux central), dont le diagnostic repose sur une prise de sang. Dans la Vienne, sur les 22.000 enfants de moins de 6 ans, 29\% résident dans un logement construit antérieurement à 1948 (INSEE). Selon l'INSERM, $2 \%$ de la population de 1 à 6 ans a une plombémie supérieure à $100 \mu \mathrm{g} / \mathrm{l}$, soit 350 cas potentiels dans le département. Les modes d'exposition sont 
bien connus : habitat ancien dégradé contenant des peintures au plomb, travaux sans précaution sur revêtements anciens, adoption internationale, hygiène et activité de ferraillage, activité professionnelle du père, usages à risques (bijou, khôl, vaisselle importée, kéfir, etc.). Suite à plusieurs dispositifs législatifs mis en place depuis 1998 au niveau national ${ }^{1}$, a été mis en place dans la Vienne, un dépistage des populations à risque par Médecins du Monde, et, depuis 2000, des missions de repérage du plomb dans l'habitat (en particulier dans le parc OPAC). Ces actions ont été suivies de la mise en place d'un Comité Technique Plomb (2002) qui a effectué un zonage (ERAP) ; enfin, à partir de 2004 s'est mise en place une campagne de dépistage par l'habitat (campagne de Châtellerault) et en 2008 un dépistage par population à risque. Depuis, on constate une forte augmentation du nombre d'enfants dépistés devant bénéficier d'une prise en charge médicale (9 plombémies $>450 \mu \mathrm{g} / \mathrm{L}$ en 2005). La prise en charge de l'enfant est donc le plus souvent limitée à son environnement, mais l'existence d'effets même à faibles doses plaide pour la prévention primaire pour tous les enfants à risque. Le dépistage reste un moyen de mettre en évidence l'existence d'un problème de santé publique et de mobiliser les acteurs de la prévention. Cet exposé a été enrichi de la projection d'extraits du documentaire « Du plomb dans la tête » (2003; réalisation Fabrizio Scapin, co-production AFVS - Fabrizio Scapin).

Enfin, Maïté Duballet (coordinatrice du ReVIH86) et Corinne Paul (assistante sociale, ReVIH86) ont exposé les actions du ReVIH86 dans la prise en charge de patients

\footnotetext{
${ }^{1}$ La loi du 29 juillet 1998 relative à la lutte contre l'exclusion, suite à la loi du 9 août 2004 relative à la politique de santé publique et plus précisément aux arrêtés du 25 Avril 2006 relatifs au diagnostic du risque d'intoxication par le plomb des peintures, au contrôle des travaux en présence de plomb, au constat de risque d'exposition au plomb et aux travaux en parties communes nécessitant l'établissement d'un constat de risque d'exposition au plomb.
}

atteints du VIH-SIDA dans la Vienne. Le ReVIH86 est une cellule de coordination médico-sociale pour l'aide au maintien à domicile des personnes atteintes du VIH et ou des hépatites. Le ReVIH est une association loi 1901, créée en septembre 1994, visant, comme tous les RVH, à améliorer la prise en charge médico-sociale des personnes vivant avec le VIH et/ou le VHB/VHC, à être l'interlocuteur privilégié en matière de prise en charge des malades et de formation des professionnels, et de suivi et d'évaluation du dispositif du maintien à domicile. Le personnel intervenant au ReVIH86 est composé d'une infirmière coordinatrice, d'une assistante sociale d'une secrétaire, d'un psychologue (vacations), et de bénévoles. Toutes les personnes atteintes des pathologies ciblées peuvent être prises en charge ; la file active en 2009 est de 195 personnes (dont 44 VHC), dont 34\% d'étrangers. Le ReVIH a pour partenaires privilégiés l'ensemble des acteurs intrahospitaliers (médecins, infirmières, psychologues, assistantes sociales hospitalières, diététiciennes, relais Georges charbonnier, PASS, services de soins du CHU, services de soins de l'hôpital H. Laborit, CMP, Le Tourniquet) et les acteurs extrahospitaliers (médecins généralistes, pharmacie, infirmiers libéraux, kinésithérapeutes, SSIAD, services d'aide à domicile, assistantes sociales de secteur CPAM, centres d'hébergement: CHRS, ACT, CCAS, Résidence Cécile et Marie-Anne..., associations: Croix Rouge, Sanza, Vima, Aides, Fil d'Ariane, Secours Catholique...). L'accompagnement individuel, la réponse à des besoins sociaux et médicaux spéciaux, l'attribution d'aides médico-sociales sont le lot commun de tous les RVH, mais le ReVIH86 cherche à coordonner quelques actions précises: accompagnement physique dans la recherche d'hébergement, financement de traducteurs, financement de cartes de téléphone pour maintenir le lien avec les enfants restés au pays, financements de frais d'optique et ou dentaires en compléments du droit commun, recherche de financement pour frais de délivrance du passeport et frais de transport 
jusqu'à l'ambassade du pays à Paris, financement du taux d'effort pour un logement en résidence sociale ou autres, coordination des rendez-vous médicaux, éducation thérapeutique (à adapter au domicile de la personne). Ces pathologies chroniques demandent une prise en charge médicale et para médicale spécifique, pour lesquelles la prise en compte des aspects sociaux, des conditions de vie, des effets indésirables des traitements, et de l'observance est indispensable. Les stratégies d'évolution et de réorientation des objectifs et des actions se fait largement au gré de l'arrivée d'un nouveau type de patients, avec la population migrante, et au fil des changements dans le droit à la santé en lien avec les politiques migratoires.

Cette journée aura montré qu'il existe un ensemble d'initiatives qui émanent du tissu d'acteurs locaux. En somme, les situations qui ont été décrites au cours de cette journée sont plutôt extrêmement variées au niveau local et se renouvellent en permanence devant les changements dans les politiques migratoires. Ce qui reste constant dans les débats qui ont encadré les exposés, c'est la dimension morale qui encadre la problématique générale des actions de santé à destination des migrants, les faisant alors entrer dans des débats publics plus larges, touchant aux questions de particularisme (faut-il adapter des actions en fonction de l'origine nationale des destinataires ?) et de différenciation (comment prendre en charge les populations marginales et vulnérables qui on un accès différencié à la santé et aux soins). Plusieurs enjeux ont ainsi pu être repérés. Le premier se rapporte à la qualification des publics et à la spécification des actions: comment qualifier et repérer la population cible (par rapport à un comportement? à une pathologie ?). Cet enjeu aboutit sur un travail autour des valeurs projetées dans les actions concernant la santé des migrants (discrimination positive/principes d'égalité; particularisme culturel/principe d'universalité). Le deuxième enjeu relève des oscillations des actions et des acteurs entre responsabilité individuelle et participation collective.

Anne-Cécile Hoyez Chargée de recherche CNRS/ MIGRINTER - UMR 6588 anne.cecile.hoyez@,univ-poitiers.fr 